\title{
Germanica
}

\section{Anmerkungen zum Begriff Gesamtkunstwerk - die Politisierung einer ästhetischen Kategorie?}

A propos du «Gesamtkunstwerk». La politisation d'une catégorie esthétique?

Till R. Kuhnle

\section{OpenEdition}

\section{Journals}

Édition électronique

URL : http://journals.openedition.org/germanica/2085

DOI : 10.4000/germanica.2085

ISSN : 2107-0784

Éditeur

Université de Lille

\section{Édition imprimée}

Date de publication : 1 janvier 1992

Pagination : $35-50$

ISSN : 0984-2632

\section{Référence électronique}

Till R. Kuhnle, « Anmerkungen zum Begriff Gesamtkunstwerk - die Politisierung einer ästhetischen Kategorie? », Germanica [Online], 10 | 1992, Online erschienen am: 12 Februar 2014, abgerufen am 06 Oktober 2020. URL : http://journals.openedition.org/germanica/2085 ; DOI : https://doi.org/10.4000/ germanica.2085

Ce document a été généré automatiquement le 6 octobre 2020.

(C) Tous droits réservés 


\title{
Anmerkungen zum Begriff Gesamtkunstwerk - die Politisierung einer ästhetischen Kategorie?
}

\author{
A propos du «Gesamtkunstwerk». La politisation d'une catégorie esthétique?
}

\author{
Till R. Kuhnle
}

1 «Von Gesamtkunstwerken wollen wir in allen Bereichen dann sprechen, wenn Individuen ein gedankliches Konstrukt übergeordneter Zusammenhänge als bildliches System oder als politische Utopie entwickelt haben $»^{1}$. So definiert der Ästhetiker Bazon Brock in einem Artikel zu der Ausstellung Der Hang zum Gesamtkunstwerk - Europäische Utopien seit 1800 einen Begriff, der nach Ernst Bloch «nur durch Wagners Genie zu einer lebensfähigen, wenngleich prinzipiell unkanonischen Kategorie werden konnte» ${ }^{2}$. Der folgende Beitrag versucht, die Bedeutung dieser Kategorie für die moderne Ästhetik zu erhellen und die historischen Determinanten von Konzeptionen, die einer ästhetischen oder politischen Intention gehorchend die Verschmelzung der Kunstgattungen fordern, hervorzuheben. Der Idee vom Gesamtkunstwerk liegt ein Totalitätsanspruch zugrunde, wodurch sie sich der Komplizenschaft mit der Instrumentalisierung der Ästhetik im Faschismus verdächtig macht. Die «Anmerkungen zum Gesamtkunstwerk» gelten einer Kategorie, welche möglicherweise eine Brücke für die Vereinnahmung der Tradition durch eine totalitäre Ideologie darstellt. Es wird dabei nicht der Anspruch erhoben, die kontroversen Ansichten zu einer Ästhetik des Gesamtkunstwerkes zu bündeln.

2 Die Überlegungen gehen von Walter Benjamins Das Kunstwerk im Zeitalter seiner technischen Reproduzierbarkeit ${ }^{3}$ aus. Dieser Aufsatz markiert eine wichtige Etappe, wenn nicht eine Wende, in der Diskussion um die Funktionsbestimmung von Kunst im Zeichen des Faschismus. Während Walter Benjamin mit Blick auf eine kommunistische Gesellschaft an einem programmatischen Entwurf zur Verbindung von Kunst und 
Lebenspraxis arbeitet, fragt Adorno in seinem Versuch über Wagner nach dem ideologischen Gehalt von Konzeption und Praxis des Wagnerschen Musikdramas ${ }^{4}$.

\section{Das Herauslösen der Kunst aus dem Ritual oder der «Tod des Gesamtkunstwerks»}

3 In bezug auf den Traditionszusammenhang eignet dem Kunstwerk ein «je ne sais quoi ", d.h. eine diskursiv nur schwer oder gar nicht zu fassende Eigenschaft, die Benjamin die Aura genannt und als «einmalige Erscheinung einer Ferne, so nah sie sein mag» (Benjamin, Kunstwerk, S. 15) definiert hat. Die Aura eines Objekts besteht darin, daß dieses für das erkennende Subjekt eine über seinen rein empirischen Aspekt hinausweisende Eigenschaft besitzt; das aureatische objekt entzieht sich der instrumentellen Verwertung. Die ursprüngliche Erfahrung der Aura entspringt nach Benjamin dem Augenblick des kontemplativen Betrachtens eines Naturschauspiels. Auf ein Artefakt bezogen, bezeichnet die Aura eine qua Zugehörigkeit zu einer Kollektivität bestimmte Art und Weise der Wahrnehmung, zu der das religiöse Ritual mit seinen Fetischen und Götzen ebenso zählt wie ein profaner Kult des Schönen, der mit der Renaissance einsetzt und in die Resaralisierung der Kunst durch die Maxime des «l'art pour l'art» und den Ästhetizismus mündet. Die Voraussetzung für die aureatische Rezeptions weise schafft also ein Kult /ein Ritual: «Der einzige Wert des "echten" Kunstwerks hat seine Fundierung im Ritual, in dem es seinen originären und ersten Gebrauchswert hatte» (Benjamin, Kunstwerk, S. 16). Bei diesen Gebilden sei es wichtiger, daß sie vorhanden sind als daß sie gesehen werden. Mit ihrem Herauslösen aus dem Kult wachse die Gelegenheit zur Ausstellung.

4 Die entscheidende Wende erkennt Benjamin in der Entwicklung moderner Reproduktionstechniken, die in der Erfindung des kinematographischen Apparates gipfelt. Diese Techniken bedeuten eine Überwindung der für die Aura konstitutiven Distanz; das Objekt kann nahezu beliebig oft ausgestellt werden, wodurch die Aura definitiv zertrümmert wird. Problematisch bleibt Benjamins einseitige Fixierung auf den Aspekt der Reproduktion, da er die Kategorie der Aura offensichtlich nicht in ihrer Historizität erfaßt. Galt in der Totalität des Kultes, die für die aureatische Erscheinungsweise des Kunstwerkes konstitutiv war, eine kollektive Rezeption, so ist diese von der kontemplativen Betrachtung von Kunst durch das Individuum zu unterscheiden. Es sei hier in Erinnerung gerufen, daß sich «Kunst» bzw. «schöne Künste» als Oberbegriff für die verschiedenen Gattungen wie Malerei, Musik, Poesie usw. erst im Laufe des 18. Jahrhunderts herausgebildet hat ${ }^{5}$, zeitgleich also mit der wachsenden gesellschaftlichen Bedeutung des Bürgertums. Bürgerlicher Individualismus steht quer zu den kollektiven Rezeptionsformen innerhalb eines (religiösen) Kultes. Ungeachtet des hier vorgebrachten Einwands gegen Benjamin führt der Begriff Aura als analytische Kategorie die Kunsttheorie weiter, was ein Blick auf den Kult des Schönen, den "profanen Schönheitsdienst», belegt. Im Namen der Schönheit versuchte man die Distanz zum objekt wieder herzustellen, welche die aureatische Rezeptionsweise charakterisiert. Auf die nunmehr fehlende kollektive Praxis folgte die individuelle Kontemplation. Durch die neuen Reproduktionsmittel verlor das Kunstwerk definitiv seine Einmaligkeit, da es allgemein verfügbar wurde; mit seiner Vervielfältigung trat das Kunstwerk als Artefakt hervor, ja als industrielles Produkt. Der Film zeigt besonders deutlich die Tendenz hin zur «Reproduktion eines 
auf Reproduzierbarkeit angelegten Kunstwerks»(Benjamin, Kunstwerk, S. 17). Die aureatische Rezeption sowohl kollektivkultischer als auch individuellkontemplativer Art geriet in Gefahr. In diesem Kontext sind die bereits genannten Bewegungen des l'art pour l'art und des Ästhetizismus zu nennen. Es wäre jedoch zu kurz gegriffen, das Entstehen dieser Bewegungen allein auf die Reproduzierbarkeit des Kunstwerks zu beziehen; vielmehr scheint sich hinter dem, was Benjamin als Verlust der Aura bezeichnet hat, noch eine weitere, nicht allein im Bereich des Ästhetischen angesiedelte Leerstelle auf zutun. Das vor allem im 19. Jahrhundert sich deutlich manifestierende Unbehagen an dem Ausdifferenzieren der verschiedenen Kunstgattungen verrät ein Streben nach jener Erfahrung von Totalität, die in der Lebenspraxis nicht mehr zu verwirklichen ist; in anderen Worten: das Verschmelzen der Künste verheißt jenseits der Lebenspraxis ein Aufheben der Entfremdung ${ }^{6}$.

Vom «Tod des Gesamtkunstwerks» und dem Entstehen einer «musealen Situation» spricht der konservative Kunsttheoretiker Hans Sedlmayr ${ }^{7}$ und versteht darunter die im 18. Jahrhundert einsetzende Verlagerung der Kunstwerke aus den Kirchen und Schlössern in die Museen. Die Rede vom «Tod des Gesamtkunstwerks» verrät, daß der status quo ante, d.h. vor dem Ausdifferenzieren der Künste, vom Ästhetischen her betrachtet wird.

\section{Sulzer und Schelling über das Zusammen wirken der Künste}

6 Der Oper, in der das Zusammenwirken der Einzelkünste praktiziert wird, standen deutsche Ästhetiker seit dem ausgehenden 18. Jahrhundert kritisch gegenüber. Sulzer erkannte in der Oper ein Gebilde, in dem die Einzelkünste nur verkümmert seien. Unter bestimmten Voraussetzungen aber konzedierte Sulzer der Oper eine gelungene Wirkung:

Wenn man bedenkt, was für große Kraft in den Werken einer einzigen der schönen Künste liegt, wie sehr der Dichter uns durch eine Ode hinreißen, wie tief uns der Tonsetzer auch ohne Worte rühren, was für lebhafte und dauernde Eindrücke der Maler auf uns machen kann; wenn man zu allem diesem noch hinzusetzt, daß das Schauspiel schon an sich die Empfindungen auf den höchsten Grad treibet: So wird man begreifen, wie unwiderstehlich die Gemüter der Menschen durch ein Schauspiel konnten hingerissen werden, in welchem die einzelnen Kräfte der verschiedenen schönen Künste so genau vereinigt sind. Ich stelle mir vor, daß bei einer wichtigen Feierlichkeit; z.B. bei der Thronbesteigung eines Monarchen, eine in allen Teilen wohl angeordnete und gut ausgeführte Oper gespielt würde, die darauf abzielt, neue Fürsten empfinden zu lassen, was für ein Glanz den Regenten umgibt $[. . .]^{8}$.

7 Hier tritt offenkundig ein außerästhetischer Faktor hinzu, welcher die Kunstgattungen einem gemeinsamen Prinzip unterordnet: die Politik. Benjamin hat davon gesprochen, $\mathrm{da}$ an die Stelle der Fundierung der Kunst auf das Ritual ihre Fundierurig auf die Politik trete. Das einseitige Festhalten am Reproduktionsparadigma verstellt ihm hier aber den Blick auf die von der politischen Veränderung im 18. Jahrhundert bereits ausgehende explizite Indienstnahme der Kunst durch die Politik.

Ebenfalls auf den Bereich des Außerästhetischen verwies Schelling, als er 1802 in seiner Philosophie der Kunst über «die vollkommenste Zusammensetzung aller Künste» im Drama des Altertums, «wovon uns nur eine Karikatur, die Oper, geblieben» sei, schrieb: 
Musik, Gesang, Tanz, wie alle Arten des Dramas leben selbst nur im öffentlichen Leben und verbünden sich in diesem. Wo dieses verschwindet, kann statt des realen und äußeren Dramas, an dem, in allen seinen Formen, das ganze Volk, als politische oder sittliche Totalität, Teil nimmt, ein innerliches, ideales Drama allein noch das Volk vereinigen. Dieses ideale Drama ist der Gottesdienst, die einzige Art wahrhaft öffentlicher Handlung, die der neueren Zeit, und auch dieser späterhin nur sehr geschmälert und beengt geblieben ist ${ }^{9}$.

Beide Autoren, Sulzer und Schelling, setzen eine kollektive Rezeption dieser Synthesen aus den verschiedenen Kunstgattungen voraus; das Unbehagen an Vereinzelung und Innerlichkeit kündigte sich an, das Nietzsches Philosophieren bestimmen sollte.

Mit dem theoretischen Durchdringen der in Gattungen ausdifferenzierten Künste entstand der Gedanke an ihr Verschmelzen zu einem einzigen Kunstwerk. Für Schelling bestand die Absolutheit der Kunst darin, «daß das Allgemeine der Kunst und das Besondere, welches sie im Künstler als Individuum annimmt, absolut eins, dieses Besondere das ganze Allgemeine sei, und umgekehrt» (Schelling, Philosophie der Kunst, S. 302). Die Kategorie des Kunst-Werks beruht auf der Annahme einer Einheit von Allgemeinem und Besonderem ${ }^{10}$. Der Gedanke an die Verschmelzung der Künste hält an dieser Kategorie fest. Nur unvollkommen vereint, repräsentieren die Gebilde der jeweiligen Gattung nicht mehr allein jene Einheit von Allgemeinem und Besonderem, wodurch sie sich als Kunstwerk auszeichnen. Der Anspruch der Kunst auf Absolutheit muß nun durch das Zusammenwirken der Künste erfüllt werden, womit die Ästhetik an ihre Grenzen stößt. Schellings Schrift erkennt in einer religiös fundierten Totalität den Garanten einer gelungenen Vereinigung der Künste. In einer anderen Schrift des Philosophen heißt es: «Wenn es auch nicht allgemein eingesehen werden könnte, daß die Kunst ein notwendiger und integranter Teil einer nach Ideen entworfenen Staatsverfassung ist, so müßte wenigstens das Altertum daran erinnern, dessen allgemeine Feste, verewigenden Denkmäler, Schauspiele, so wie alle Handlungen des öffentlichen Lebens nur verschiedene Zweige Eines allgemeinen objektiven und lebendigen Kunstwerks waren» ${ }^{11}$.

\section{Propaganda und Verdrängung}

Konzeptionen zur politischen Instrumentalisierung der Künste, wobei die Idee ihrer Verschmelzung durchaus im Mittelpunkt steht, sind in Frankreich seit der französischen Revolution entstanden. Es würde den Rahmen dieser Abhandlung sprengen, wollte man die Geschichte der Kunst der französischen Revolution hier aufarbeiten. Ein Blick auf eine politische Bewegung, deren Entstehung in die Epoche der französischen Romantik fällt, sei dafür gestattet.

Frühsozialistische Theoretiker aus der Schule Saint-Simons wiesen den Künstlern und den von ihnen vertretenen Gattungen eine führende Rolle in ihrer utopischen Gesellschaftsordnung $\mathrm{zu}^{12}$ : Künstlerisches Schaffen soll in dem Kultus einer panentheistischen Kunstreligion ${ }^{13}$ aufgehen, die das gesellschaftliche Leben als eine geschlossene Totalität faßt. Ästhetische Manifestation und industrielle Produktion gehen bruchlos ineinander über; die Batterie wird zu einer modernen Kathedrale ${ }^{14}$.

Der Saint-Simonist Emile Barrault stellte in seinem Appel aux artistes einen Entwurf der Kunst der Zukunft vor ${ }^{15}$. Die Künste allgemein sah Barrault in einem einzigen Kult miteinander vereinigt, wobei der Rhetorik der Rang zufallen sollte, die erste unter den 
Künsten zu sein: «Enfin la musique, la peinture, la sculpture seconderont les efforts de l'éloquence et de la poésie dans les temples que l'architecture aura renouvelés sous l'inspiration d'un culte nouveau, ralliant tous les hommes au pied des mêmes autels, aura paru sur la terre » (Barrault, S. 84) ${ }^{16}$.

Barraults Ausführungen zur Geschichte der Kunst und zum Entwurf eines gigantischen Kultes erschien nur 3 Jahre nach Victor Hugos Préface de Crom well. Hugos nach Monumentalität strebendes « drame », die Vollendung einer " poésie complète », ist ein die Grenzen einer Gattung sprengendes Prinzip, das in der Schauspielkunst zu walten habe, eine regelrechte "AntiGattung». Ähnlich Hugo gelang es Barrault nicht, seine Vorstellungen von dem zukünftigen Wirken der Künste in den Kategorien tradierter Gattungen auszudrücken. Während Hugo als Dramenautor das Theater problematisierte, zeichnete der saint-simonistische Kunsttheoretiker Barrault das Bild von einer kultischen Vereinigung der Künste zu einem Ganzen, das wir als das saintsimonistische «Gesamtkunstwerk» ${ }^{17}$ bezeichnen wollen.

Eingangs ist die Frage aufgeworfen worden, ob der Hang zum Gesamtkunstwerk nicht einer Leerstelle entspringt, der die kontemplative Rezeption von isolierten Kunstwerken nichts entgegenzusetzen hat. Ein Blick auf das Theater - man hätte ebensogut die Architektur heranziehen können - mag dies verdeutlichen. Benjamins Kritik etwa an der bürgerlichen Auffassung vom Theater trifft den ideologischen Kern einer Gattung, die über ihre Grenzen hinausstrebt. In ihr manifestiert sich das Selbstbewußtsein der Bourgeoisie und ihr Glaube an das Machbare in der Welt. Zugleich verschleiert das im bürgerlichen Kulturbetrieb etablierte Theater mit seinem versöhnlichen Gestus die tatsächlichen Herr schafts Verhältnisse. Der Theaterabend blendet die sozialen Konflikte aus. Dieser rückschrittlichen Auffassung vom Theater «ist und bleibt die Harmonie des ganzen ungetrübt und der Mensch ist ihr Repräsentant. Sie sieht ihn auf der Höhe seiner Macht, als Herrn der Schöpfung, als Persönlichkeit. (Und wäre er der letzte Lohnarbeiter)» ${ }^{18}$.

Das großbürgerliche Theater, das sich als «Symbol», «Totalität» oder «Gesamtkunstwerk» realisiere (Benjamin, Brecht, S. 98), hat in seinen Effekten mit dem Film zu wetteifern begonnen - ein klägliches Unterfangen. Kritisches Theater wie das von Brecht aber, das die Effekthascherei durchbricht, bietet neue Möglichkeiten, dem Menschen den Blick auf die eigene Realität zu öffnen: «Das epische Theater stellt dem dramatischen Gesamtkunstwerk das dramatische Laboratorium gegenüber» (Benjamin, Brecht, S. 99). Eine Theaterkonzeption, der wir hier ohne weitere Präzisierung mit Benjamin das Etikett «Gesamtkunstwerk» angeheftet haben, entstand zeitgleich mit einer Reihe von Attraktionen des 19. Jahrhunderts: Dioramen, Panoramen und Nocturamen versprachen, den romantischen Traum vom Zusammenwirken der Künste zu verwirklichen. Der Film ${ }^{19}$ - lediglich «eine Natur zweiten Grades» (Benjamin, Kunstwerk, S. 31) - wiederum strebt nach einer Perfektion, welche die Trennung von Zuschauerraum und Filmgeschehen durch optische Kniffe aufzuheben sucht. Die Leinwand verliert ihre Funktion als «Trennwand»: Dreidimensionales Kino und mehr noch die Géode im Pariser Parc de la Villette sind Ausdruck dieser im Zeichen der Postmoderne noch immer beharrlichen Tendenz. 


\section{Das «Gesamtkunstwerk» - ein mißbrauchter Begriff?}

Die Einführung der Kategorie Gesamtkunstwerk, die in der Kulturgeschichte untrennbar mit dem Namen Richard Wagner verbunden ist, erfordert nunmehr eine Präzisierung. Richard Wagner knüpfte 1849 in einer programmatischen Schrift an die griechische Tragödie an, die ihm als Vorbild für sein «Drama», das «Kunstwerk der Zukunft», diente:

Das große Gesamtkunstwerk, das alle Gattungen der Kunst zu umfassen hat, um jede einzelne dieser Gattungen als Mittel gewissermaßen zu verbrauchen, zu vernichten zu Gunsten der Erreichung des Gesamtzwecks aller, nämlich der unbedingten, unmittelbaren Darstellung der vollendeten menschlichen Natur, dieses große Gesamtkunstwerk erkennt er [sc.: der Geist] nicht als die willkürlich mögliche Tat des Einzelnen, sondern als das notwendig denkbare gemeinsame Werk der Menschen der Zukunft ${ }^{20}$.

Adorno hat die äußere Erscheinungsform des Wagnerschen Gesamtkunstwerks als ein «quid pro quo ästhetischer Medien [...], welches durch artifizielle Vollendung alle Nahtstellen des Artefakts, ja dessen Differenz von der Natur selber verdecken soll» (Adorno, Wagner, S. 90), charakterisiert. Die Abneigung von Sulzer und Schelling gegen die Oper rührt gerade daher, daß in ihr das Artefaktische bar jeden Anspruchs auf Absolutheit in der nur unvollkommenen Darstellung zum Vorschein kommt, wenn es nicht gelingt, die Künste zu einem vollkommenen «Werk» zu bündeln; sie fürchteten den Schaden, den die jeweilige Einzelgattung in dem unvollkommenen Zusammenwirken der Künste nehmen könnte. Darauf antwortete Wagner, daß die einzelne Gattung als Mittel «verbraucht» werde. Sie muß also ihre Identität leugnen.

Wagner hat sich in späteren Schriften gegen den Mißbrauch seines Begriffs «Gesamtkunstwerk» verwahrt; wir bekennen uns dagegen zu diesem Mißbrauch und fassen den Begriff des Gesamtkunstwerks weiter: Unter Gesamtkunstwerk wollen wir ein ästhetisches Gebilde verstehen, das die Mittel akustischer, bildender und darstellender Künste dergestalt zu einem einheitlichen Ganzen vereint, daß weder die Einzelkünste die Möglichkeit zur Entfaltung einer dialektischen Beziehung von Form und Inhalt erhalten, die mit dem isolierten Gebilde einer Gattung gegeben wäre, noch aber die Antithetik der in ihm enthaltenen. Kunstmaterialien produktiv eingesetzt wird. Die im Gesamt kunstwerk vereinten Künste werden einzig auf einen bestimmten Effekt hin ausgerichtet; dieser beruht «nur auf empirischtechnischen Regeln» (Schelling, Philosophie der Kunst, S. 564). Der Effekt kann sich auf die Erzeugung der Illusion von Totalität in der rauschhaften Vereiningung der Künste beschränken - so das Wagnersche Musikdrama - oder aber in den Dienst eines primär außerästhetischen Zwecks gestellt werden - so in den propagandistischen Inszenierungen totalitärer Provenienz. Während das Gesamtkunstwerk in Gestalt des Wagnerschen Musikdramas - entgegen Wagners eigenem Anspruch - eine ins äußerste getriebene Ausformung autonomer Kunst bedeutet und sich selbst in Frage zu stellen droht, dient die Vermischung der Kunstgattungen zum Zwecke totalitärer Propaganda der Umdeutung der Wirklichkeit, indem diese mit der Aura einer Vollkommenheit umgeben wird, die sie nicht haben kann. Folgerichtig weisen die Anhänger Wagners den Vorwurf des Dilettantismus gegen ihr Idol zurück ${ }^{21}$ bzw. verzichten auf eine positive Besetzung des Begriffs, da dieser das Eingeständnis in die Teilbarkeit und damit virtuelle Eigenständigkeit der nunmehr wieder zueinander findenden Künste bedeuten würde. Zugleich hält Wagners Künstler der Zukunft an der Werkkategorie fest: Das 
Musikdrama ist als Gesamt-Kunstwerk angelegt; es soll - ganz im Sinne Schellings - das Absolute repräsentieren. Idem es aber den Einzelgattungen die Fähigkeit abspricht, dieses Absolute im Kunstwerk zu realisieren, negiert es gerade die Kategorie, der es seine Existenz verdankt. Die so entstandene Aporie vermag nur dahingehend aufgelöst zu werden, daß jeglicher dialektische Bezug zur Lebenspraxis geleugnet, ja diese dem Gesamtkunstwerk einverleibt wird. Die Grenze zwischen Ästhetik und (totalitärer) Gesellschaftsutopie ist durchbrochen. Hier gewinnt das von Benjamin eingeführte Reproduktionsparadigma wieder an Gewicht. In der Tat ist das Gesamtkunstwerk an das hic et nunc seiner Realisierung gebunden. Die Reproduzierbarkeit macht es in diesem Punkt verwundbar. Das soll nicht heißen, daß die Kategorie des Gesamtkunstwerks angesichts modernster Medien wie ein Kartenhaus zusammenbricht; von ihrem Anspruch her verweist sie über die jeweilige Repräsentation hinaus. Das Gesamtkunstwerk findet sich aber in der Praxis immer nur als sein eigener Schein verwirklicht.

Jede Untersuchung zum Phänomen Gesamtkunstwerk sieht sich mit einer Frage konfrontiert: Wie verhält sich die Kunst zur Lebenspraxis? Im bürgerlichen Theaterverständnis, das seit etwa der 2. Hälfte des 19. Jahrhunderts von Wagners Ideen entscheidend geprägt war, bildet sich das Theater zu einem Ort heraus, in dem eine Lebenspraxis vorexerziert wird, die einlöst, was die Realität verweigert. Diese Position bildet nur einen scheinbaren Gegensatz zu einer weiteren, die den Primat der gesellschaftlichen Utopie für die Bestimmung der Künste festschreibt. Das Ästhetische hebt in einem der Lebenspraxis fernen Bereich deren Widersprüche auf. Hier setzt eine andere Konzeption an, welche die Kunst bzw. das Ästhetische in die Politik hineinzutragen sucht. Sie gibt dabei vor, die gesellschaftlichen Widersprüche nunmehr in der Lebenspraxis selbst aufgehoben zu haben. Bayreuth hat auf seine Weise im scheinbar gesellschaftsfreien Raum diese Aporie am Wagnerschen Musikdrama vorgelebt: der Gedanke einer letztlich vom Ästhetischen her organisierten Gesellschaft und das gleichzeitige Zelebrieren der eigenen Autonomie als «Kunstwerk». Das Musikdrama drängt über den Festspielort hinaus und bildet einen Höhepunkt jenes Fetischismus, der den Hochkapitalismus charakterisiert (Marx, MEW 23, S. 86 ff): Das Artefakt leugnet seine Herkunft aus der menschlichen Arbeit und verselbständigt sich zu einem magischen Gebilde. Die Erfahrung der «einmaligen Erscheinung einer Ferne, so nah sie auch sein mag» (Benjamin) scheint wieder wirklich geworden, strebt doch «das Gesamtkunstwerk dem Ideal des absoluten Phänomens nach» (Adorno, Wagner, S. 91). Folgerichtig leugnet Wagner das Prinzip der Arbeitsteilung, wenn er vom Willen einer jeden Kunstart spricht, in einem einzigen, übergeordneten Kunstwerk aufzugehen: «Wenn sie [...] ganz einer anderen sich gibt, so bleibt sie auch ganz in ihr erhalten, vermag ganz aus ihr in die dritte überzugehen, um so im gemeinsamen Kunstwerk in höchster Fülle ganz sich selbst wiederum zu sein» (Wagner, Werke, VI).

\section{Das Gesamtkunstwerk im Zeichen der «Ästhetisierung des politischen Lebens» durch den Faschismus}

21 Adorno hat darauf hingewiesen, was Wagner in seinem ästhetischen Anspruch vorexerziert: "Theoretisch und dann in der Ideologie der Werke hat er die Arbeitsteilung abgelehnt mit Parolen, die an die nationalisozialistischen von der Überwindung der Sonderinteressen durch den Gemeinnutz gemahnen» (Adorno, 
Wagner, S. 101). Damit haben wir eines der Essentials faschistischer Ideologie, das Benjamin mit seinem Diktum von der «Ästhetisierung des politischen Lebens» (Kunstwerk, S. 42 ff) auf den Punkt gebracht hat. Die faschistische Ideologie nimmt die Stelle jenes übergeordneten Prinzips an, das Wagner als conditio sine qua non des vollendeten Zusammenwirkens der Künste genannt hat: «Das Kunstwerk ist die lebendig dargestellte Religion; - Religionen aber erfindet nicht der Künstler, sie entstehen aus dem Volk» (Wagner, Werke VI, S. 31). Der Lichtdom auf dem Nürnberger Parteitag und andere propagandistische Veranstaltungen der Nationalsozialisten sind ein beredtes Zeugnis dafür ${ }^{22}$, mehr noch der totale Krieg mit seinen Stahlgewittern, der dazu auserkoren war, jeden einzelnen «gewissermaßen als Mittel zu verbrauchen». Der Krieg ermöglicht die Hypostasierung der Erfahrung, daß des Mensch seine Bestimmung im Untergang findet. Die Apologie des Krieges setzt die Ästhetik perfide dazu ein, diesem den Schein von Notwendigkeit zu geben, den die Entwicklung modernster Kriegstechnologie aufrechtzuerhalten hilft. Wir geben an dieser Stelle zu bedenken, was Schelling zur Architektur über die conditio sine qua non für ihre Konstituierung als Kunst festgestellt hat: «Es muß also eine innigere, scheinbar aus dem Objekt selbst kommende Identität, eine wahre Verschmelzung mit dem Begriff sein, was die Architektur zur schönen Kunst macht. Bei dem bloß mechanischen Kunstwerk ist dieser Zusammenhang immer nur subjektiv» (Schelling, Philosophie der Kunst, S. 406). Der Faschismus erweckt den Schein dieser Identität von Objekt und Begriff, indem er sich - wohl nicht zuletzt vermittelt durch die Kategorie «Gesamtkunstwerk»Elemente einer tradierten Kunsttheorie aneignet und in die politische Praxis umsetzt.

Die Analyse politischer Utopien des 19. Jahrhunderts und totalitaristischer Ideologien des 20. Jahrhunderts haben gezeigt, daß die Entwürfe neuer Gesellschaftsordnungen mit Gesamtkunstwerkskonzeptionen in Verbindung gebracht werden können. Walter Benjamin hat den Faschismus als die «Ästhetisierung des politischen Lebens» definiert. Ästhetische Erfahrung bezeichnet hier die Selbstentfremdung in einer Gesellschaftsordnung, welche undialektisch ihre Widersprüche in einer fragwürdigen Synthesis aufhebt: Eine falsche Totalität, die nicht die geringste Abweichung erlauben und die Widersprüche durchscheinen lassen kann, ohne ihren Absolutheitsanspruch einzubüßen. Ihr fragiles Gebäude hat letztlich nur Bestand, wenn Krieg und Tod zum Telos der Kolletivität erhoben werden und so den Anspruch des in der politischen Praxis objektivierten Absoluten besiegeln. Nicht die Vernichtung des Individuellen sondern des Gesamten heißt der Einsatz: Das Alles-oder-Nichts wird zum Garanten des Absoluten, an dem der Einzelne mit der Bereitschaft, sein Leben zu opfern, Teil hat. Dem hält Benjamin die «Politisierung der Kunst» als Antwort des Kommunismus entgegen. Benjamin - und hier ist er geprägt von den historischen Avantgardebewegungen - fordert ein Durchbrechen der Trennung von Lebenspraxis und Kunst. Gesamtkunstwerksphantasien faschistischer Provenienz ordnen die Gesellschaft nach ästhetischen Prinzipien, indem sie die von einer autonomen Kunst angebotenen Konstituenten einer scheinhaften Totalität in die Lebenspraxis umsetzen. Benjamins Gegenentwurf einer Politisierung der Kunst mag heute vor dem Hintergrund des Zusammenbruchs kommunistischer Regime mit ihrer normativen Staatskunst verwundern. Kommunismus heißt für den Ästhetiker Benjamin aber - und hierin liegt der Unterschied $\mathrm{zu}$ der in den «realsozialistischen» Gesellschaften entwickelten Kunstdoktrin begründet -, daß die Kunst kritischer Reflex der Lebenspraxis ist, und dieser im Prozeß gesellschaftlicher Veränderung seinen Niederschlag findet. Die Totalität des Dialektikers ersetzt die Phantasmagorie. 
23 An Adorno anküpfend, läßt sich der Begriff «Gesamtkunstwerk» zu einer wichtigen Kategorie ideologiekritischer Kunstinterpretation ausbauen, die es auch erlaubt, ästhetische Gebilde der Moderne und Postmoderne zu hinterfragen ${ }^{23}$. Das von Wagner mit seiner Konzeption eines Gesamtkunstwerks zur Kategorie erhobene Zusammenwirken der Kunstgattungen legt ein beredtes Zeugnis von der Gefahr der Besetzung ästhetischer Begriffe durch totalitäre Ideologien ab. Benjamin hat im Vorwort zu seinem Kunst werk-Aufsatz für die Kunsttheorie eine neue, nicht durch den Faschismus korrumpierte Begrifflichkeit angekündigt:

Die im folgenden neu in die Kunsttheorie eingeführten Begriffe unterscheiden sich von geläufigeren dadurch, daß sie für die Zwecke des Faschismus vollkommen unbrauchbar sind. Dagegen sind sie zur Formulierung revolutionärer Forderungen in der Kunstpolitik brauchbar (Benjamin, Kunstwerk, S. 9).

\section{NOTES}

1. - Brock, Bazon. Der Hang zum Gesamtkunstwerk, in: Szeemann, Harald (Hg.). Der Hang zum Gesamtkunstwerk - Europäische Utopien seit 1800, 2. Aufl. Frankfurt/M, 1983, S. 22-29, hier S. 23.

2. - Bloch, Ernst. Geist der Utopie. Zweite Fassung, Frankfurt/M. 1985, S. 100.

3. - Benjamin, Walter. Das Kunstwerk im Zeitalter seiner technischen Reproduzierbarkeit, Frankfurt/ M.: Surhrkamp (ed.) 1977 (Erstv. 1936/55).

4. - Adorno, Theodor. W. Versuch über Wagner, Frankfurt/M.: Suhrkamp (st) 1981 (Erstv. 1952).

5. - Vgl. dazu die Einführung von H. Kuhn, in: Killy, Walter (Hg.). Fischer-Lexikon: Literatur II, 1, Frankfurt/M.: Fischer 1965, Artikel: «Ästhetik».

6. - Jürgen Habermas hat u.a. Nietzsches Wagner-Rezeption dahingehend gedeutet: Der Eintritt in die Postmoderne, in : Merkur 1983, S. 752-761.

7. - Sedlmayr, Hans. Verlust der Mitte, Frankfurt/Berlin/Wien 1985, S $87 f$.

8. - Sulzer, Johann Georg. Allgemeine Theorie der schönen Künste, Dritter Teil, Leipzig 1793, Artikel «Oper», S. 583f.

9. - Schelling, F.W.J. Philosophie der Kunst (1802-1803), in: Ausgewählte Schriften II, Frankfurt/M.: Suhrkamp (stw) 1985, S. 564.

10. - Vgl. dazu Bürger, Peter. Theorie der Avantgarde, Frankfurt/M.: 1974, S. 76-92.

11. - Schelling, F.W.J. Über Wissenschaft der Kunst in bezug auf das akademische Studium, in: Ausgewählte Schriften II, S. 577.

12. - So ist in dem vermutlich von O. Rodrigues verfaßten Dialog L'Artiste, Le Savant et l'Industriel erstmals von der Avantgarde-Funktion des Künstlers die Rede: Euvres de Claude-Henri de SaintSimon V, Paris : Anthropos 1966, S. 210.

13. - «Panentheismus» hat der Philosoph Karl Chr. F. Krause sein religionsphilosophisches System genannt, nach dem Gott und Welt nicht identisch sind, sondern die Welt in Gott beschlossen ist, der sie überragt; es ist die Verbindung von Theismus und Pantheismus.

14. - Duveyrier, Ch. La Ville nouvelle ou le Paris des saint-simoniens, in: Le Diable boiteux à Paris où le livre des Cent-et-un VII, Paris, 1831-1834.

15. - Barrault, Emile. Aux artistes - Du passé et de l'avenir des beaux-arts, Paris 1830.

16. - Benjamin, Walter. Das Passagenwerk, Frankfurt/M.: Suhrkamp (ed.), S. 740: «Barrault hat schon eine vage Vorstellung von der Bedeutung säkularisierter kultischer Elemente für die 
Kunst, wiewohl er den Akzent auf die kultisch gebundenen Epochen setzt». Barrault spricht übrigens auch die Rolle der technischen Reproduktion von Kunstwerken (Lithographie) für ihren Funktionswandel an.

17. - Den Begriff «Gesamtkunstwerk» hat Benjamin auf die Kunstkonzeption Saint-Simons angewandt: Passagenwerk, S. 718.

18. - Benjamin, Walter. Versuche über Brecht, Frankfurt/W.: Suhrkamp (ed) 1981, S. 98.

19. - Die Frage Der Film - Richard Wagners "Kunstwerk der Zukunft"? verneint der Musikologe Norbert J. Schneider, in: Richard Wagner und..., Regensburg 1983, S. 123-150. Schneider orientiert seinen Begriff des «Gesamtkunstwerks» strikt an der Definition Wagners.

20. - Wagner, Richard. Das Kunstwerk der Zukunft, in: Ders. Gesammelte Werke VI, Frankfurt/M.: Insel 1983, S. 28f.

21. - So Nietzsche: «Ihn (Wagner) schränkte keine strenge erb- und familienhafte Kunstausübung ein: die Malerei, die Dichtkunst, die Schauspielerei, die Musik kamen ihm so nahe als die gelehrtenhafte Erziehung und Zunft; wer oberflächlich hinblickte mochte meinen, er sei zum Dilettantisieren geboren» (Richard Wagner in Bayreuth (1876), in: Unzeitgemäße Betrachtungen, München: Goldman 1984, S. 229).

22. - Zu diesem Thema vgl. Friedländer, Saul. Kitsch und Tod. Der Widerschein des Nazismus, München: dtv 1986 und Reichel, Peter. Der schöne Schein des Faschismus, München: Hanser 1991.

23. - Vgl. a. Adorno, Th. W. Die Kunst und die Künste, in: Ders. Ohne Leitbild. Parva Aesthetica, Frankfurt/M. : Surhkamp (ed) 1967, S. 168-191.

\section{RÉSUMÉS}

Ernst Bloch sah durch Wagners Genie das Vermischen der Künste, das «Gesamtkunstwerk», in den Rang einer ästhetischen Kategorie erhoben. Die Anwendung von Walter Benjamins Kunsttheorie (Das Kunstwerk im Zeitalter seiner technischen Reproduzierbarkeit) und Theodor W. Adornos Analyse des Wagnerschen Euvres (Versuch über Wagner) auf die verschiedenen programmatischen Vorstellungen von einem Zusammenwirken der Künste erlaubt die Vermutung, daß die Kategorie Gesamtkunstwerk dazu beiträgt, das Vereinnahmen von Kunst durch totalitäre Ideologien zu fassen. Der Weg, den die Künste von ihrer Ausdifferenzierung bis zum Aufkommen der Idee vom Gesamtkunstwerk durchschritten haben, soll - nicht ohne Vorbehalt - durch Benjamins Begriff der Aura nachgezeichnet werden. Die Aura, die Benjamin als «einmalige Erscheinung einer Ferne, so nah sie sein mag» definiert hat, ist der Garant für die Echtheit und Einzigartigkeit des Kunstwerks. Diese Distanz des aureatischen Kunstwerks beruht auf seiner Fundierung im Ritual, die in dem profanen Kult des Schönen weiterlebt. Die technische Reproduktion eines Kunstwerks aber hat seine aureatische Erscheinungsweise zerstört. Dieses Phänomen ist untrennbar mit der seit dem Einsetzen der industriellen Produktionsweise und dem Entstehen der bürgerlichen Gesellschaft sich zunehmend manifestierenden Entfremdung verbunden.

Eine Ästhetik des Gesamtkunstwerks bildet die Grundlage für eine trügerische Renaissance aureatischer Rezeptions weise. Die Kunst wendet dabei das Prinzip der Arbeitsteilung an und leugnet es zugleich im vollendeten Werk. Im Gesamtkunstwerk werde, so Wagner, die einzelne Kunstgattung als Mittel «verbraucht». In dieser Apologie des Gesamtkunstwerks erkennt Adorno die Vorwegnahme eines Grundelements faschistischer Ideologie: das Partikulare soll dem Ganzen geopfert werden. 
La notion de «Gesamtkunstwerk» désigne la fusion des arts (le théâtre, la musique, la poésie, etc.) dans une œuvre qui se constitue en totalité (signifiante). D'après Ernst Bloch, c'est à partir de Richard Wagner et de sa conception du «Musikdrama» que cette synthèse s'est transformée en catégorie esthétique. En appliquant les analyses de Walter Benjamin (L'CEuvre d'art à l'époque de sa reproduction mécanisée) et de Theodor W. Adorno (Versuch über Wagner) aux différentes théories d'une synthèse des arts on peut se demander, si ce n'est pas par le biais d'une telle conception qu'une idéologie totalitaire pourrait s'emparer de l'esthétique. La description du chemin parcouru de la différenciation des arts jusqu'à leur fusion dans une œuvre qui se constitue en totalité permet - non sans réserve - l'application de la notion d'aura, par laquelle Benjamin désigne l'authenticité de l'œuvre d'art : «Une singulière trame de temps et d'espace : apparition unique d'un lointain, si proche soit-il » (L'CEuvre d'art...). Cette distance créée par l'unicité de l'œuvre d'art authentique repose sur sa fonction rituelle. Hors d'un rituel religieux, cette fonction de l'œuvre d'art persiste dans le culte profane de la beauté. Cependant, la reproduction mécanisée de l'œuvre d'art a détruit son aura, mettant ainsi en cause son authenticité. Ce phénomène est indissociable de l'aliénation croissante vécue depuis l'avènement de la production industrielle et de la société bourgeoise.

Une esthétique de l'œuvre d'art se constituant en totalité (signifiante) permet la restitution illusoire de il aura tout en niant dans le domaine de l'art ce qui caractérise la production industrielle : la division du travail. Wagner proclama l'effacement de l'individualité des arts au profit de cette totalité. Dans cette apologie wagnérienne du «Gesamtkunstwerk», Adorno découvre l'anticipation d'un des fondements de l'idéologie fasciste, qui réclame que le particulier soit sacrifié au tout.

\section{AUTEUR}

\section{TILL R. KUHNLE}

Université Charles-de-Gaulle - Lille III 